\title{
Benign and malignant (B cell) focal lymphoid aggregates in bone marrow trephines shown by means of an immunogold-silver technique
}

\author{
G SANGSTER, $\dagger$ J CROCKER,* P NAR,* MJ LEYLAND $†$ \\ From the Departments of *Histopathology and $\dagger$ Haematology, East Birmingham Hospital, Birmingham
}

SUMMARY A series of 36 iliac crest trephines, previously shown to contain follicular lymphoid aggregates, were examined using the immunogold-silver staining technique. This showed the presence of $\kappa$ and $\lambda$ surface immunoglobulin in paraffin sections of the specimens. In those trephines from patients known to be suffering from chronic lymphocytic leukaemia there was distinct monoclonality for expression of either $\kappa$ or $\lambda$ surface light chains on the lymphocyte cell surfaces in and usually between the lymphoid nodules. In specimens containing aggregates, which had previously been diagnosed as benign on morphological and clinical criteria, both $\kappa$ and $\lambda$ chains were expressed. A small number of trephines containing aggregates of uncertain nature were also examined; these all showed monoclonal expression of either $\kappa$ or $\lambda$ chains and subsequently proved to be clinically malignant. Dendritic reticulum cells, shown by staining for surface immunoglobulin, were observed only in benign aggregates.

The ability to make the histological distinction between benign and malignant infiltrates in bone marrow is clearly important. When the infiltration of a trephine specimen is both gross and diffuse it is usually possible to diagnose easily chronic lymphocytic leukaemia (CLL) or lymphocytic non-Hodgkin's lymphoma on morphological grounds alone.

Considerable histopathological difficulties often arise, however, when lymphoid cell infiltration of bone marrow trephines is focal. Such a pattern of infiltration may be the result of either marrow invasion by CLL or lymphocytic lymphoma, or may merely reflect the presence of normal benign lymphoid aggregates. ${ }^{12}$

Malignant lymphoid infiltrates should possess either $\kappa$ or $\lambda$ surface immunoglobulin $\left({ }_{s} I g\right)$ light chains, whereas the cells of benign lymphoid aggregates should be biclonal. ${ }^{3} \mathrm{Ig}$, however, is not readily shown by means of standard immunoperoxidase techniques applied to paraffin sections. To overcome this problem some workers examined ${ }_{s}$ Ig in trephine specimens by means of frozen section methods. ${ }^{4}$ The morphology attained in this way, however, is not optimal. Accordingly, we applied an immunogold-silver staining sequence to trephine specimens in an attempt to show clonality in focal lymphoid infiltrates in bone marrow trephines. Such methodology has been used for examining $\mathrm{Ig}$ in paraffin sections. ${ }^{5}$

Accepted for publication 16 December 1985

\section{Material and methods}

SPECIMENS

Thirty six iliac crest trephine biopsy samples from the same number of patients were examined having been removed by means of a Jamshidi needle. The trephines comprised 20 specimens showing focal lymphoid infiltrates in known cases of CLL. In addition, there were 12 trephines showing focal lymphoid aggregates in the absence of peripheral evidence of CLL or lymphocytic lymphoma.

These specimens were taken from patients with non-haematological malignancy or for the investigation of anaemia for which there was no evidence of leukaemia or lymphoma. Furthermore, four specimens contained focal lymphoid aggregates of unknown nature.

In the case of the known specimens of CLL surface marker studies on peripheral lymphocytes had previously confirmed the diagnosis. Conversely, peripheral cell surface markers had failed to indicate monoclonal proliferation in the cases showing benign lymphoid aggregates in their trephine biopsy specimens. In the specimens containing lymphoid aggregates of uncertain histological type peripheral cell markers were either negative or equivocal.

All biopsy specimens were taken from the routine histological files of the East Birmingham Hospital.

FIXATION AND PROCESSING

All specimens were fixed in $5 \%$ formol acetic acid- 
that is, $5 \%$ of glacial acetic acid in $10 \%$ formalin; this mixture simultaneously decalcifies and fixes and has been shown to enhance the response to immunohistological procedures in trephines. ${ }^{6}$ After fixation for 48 hours the specimens were dehydrated and taken to paraffin wax. Sections were cut at $2 \mu \mathrm{m}$ thickness, taken to aqueous media, and submitted to the following immunogold-silver staining procedure.

\section{IMMUNOGOLD-SILVER STAINING TECHNIQUE}

A standard sequence was adopted using the Janssen system (Janssen Pharmaceutica Life Sciences Products, B-2340 Beerse, Belgium). The sections were first treated with Lugol's iodine for five minutes, then rinsed in water for five minutes, and cleared with $2.5 \%$ sodium thiosulphate solution. Next, the sections were treated with $0.1 \%$ trypsin in Tris buffered saline (TBS) for five minutes, washed in TBS, then overlaid with normal (non-immune) serum diluted $1 / 5$ in TBS for five minutes. The primary antiserum (rabbit antihuman $\kappa$ or $\lambda$ light chain) was then applied at a dilution of $1 / 1000$ in TBS for 30 minutes at room temperature. After a thorough wash in TBS the sections were incubated with colloidal gold adsorbed to goat antirabbit immunoglobulin diluted $\times 1 / 500$ in TBS at $4^{\circ} \mathrm{C}$ overnight. Thorough washes in TBS (30 minutes) and distilled water (15 minutes) were performed before a wash in freshly prepared citrate

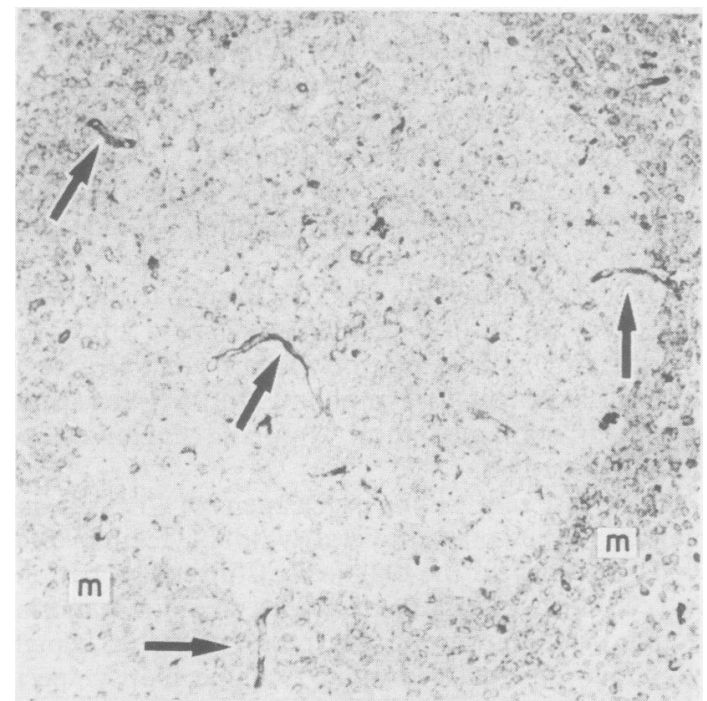

Fig. 1 Benign lymphoid aggregate, stained for $\lambda$ Ig light chains, showing well defined mantle zone (M). Occasional cells are present with strong staining for ${ }_{\mathrm{c}} \mathrm{Ig}$. In addition, cells in aggregate express ${ }_{\mathrm{s}}$ Ig. This is rather weak centrally but strong in mantle zone. Occasional blood vessels (arrows) stain for $\lambda$ chain. In addition, there is fine central meshlike activity. Immunogold-silver staining technique for $\lambda$ Ig light chain. buffer $\left(2 \cdot 35 \mathrm{~g}\right.$ trisodium citrate; $2 \mathrm{H}_{2} \mathrm{O}, 2.55 \mathrm{~g}$ citric acid, $\mathrm{H}_{2} \mathrm{O}$ in $100 \mathrm{ml}$ distilled water) for five minutes. Silver enhancement with freshly prepared solution (77 $\mathrm{mM}$ hydroquinone, $5.5 \mathrm{mM}$ silver lactate in $200 \mathrm{mM}$ citrate buffer, $\mathrm{pH} 3.85$ ) was performed under safelight conditions and the reaction stopped in $10 \%$ fixing solution (Janssen Pharmaceutica). The sections were then thoroughly washed in water, counterstained with $2 \%$ neutral red, dehydrated, cleared, and mounted in synthetic medium. The usual controls were performed.

\section{Results}

\section{BENIGN LYMPHOID AGGREGATES}

In all specimens the cells forming the aggregates were rather polymorphic. Most of the cells present were small or medium sized and rounded and corresponded to lymphocytes. In addition, many of the cells were of a similar size to the lymphocytes but possessed angulate or indentate nuclei, as they were centrocytes. In only two of the twelve specimens of benign lymphoid aggregates were secondary follicle centres observed; in these the usual cell moieties were observed-namely, centroblasts, centrocytes, and tingible body macrophages (histiocytic reticulum cells). In addition, cells were also present in these follicle centres whose nuclei corresponded to those of dendritic reticulum cells.

On staining for $\kappa$ and $\lambda_{\mathrm{s}} \mathrm{Ig}$ light chains a biclonal pattern was observed, with expression of both chains by the cells in the aggregates (Fig. 1). In addition

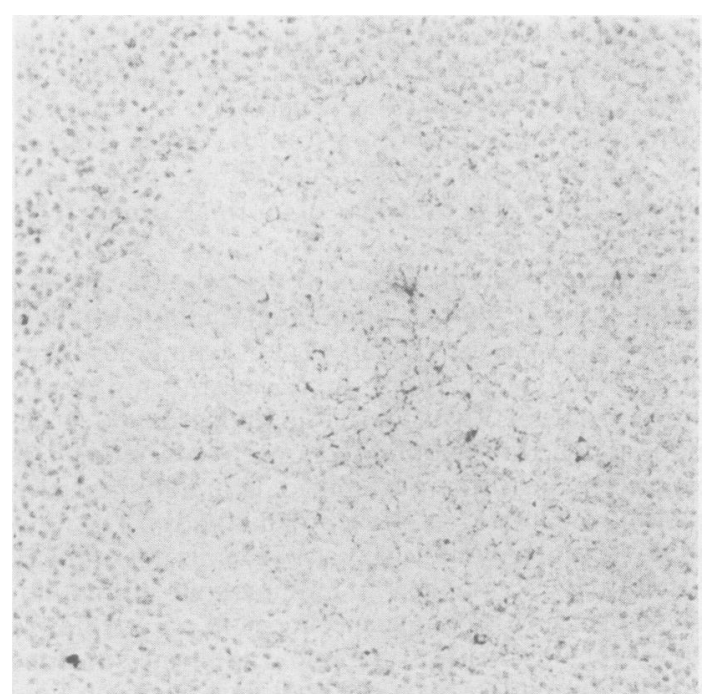

Fig. 2 Benign lymphoid aggregate lacking mantle zone. Centrally, there is fine meshlike cluster of cells, corresponding to dendritic reticulum cells. Immunogold-silver staining technique for $\kappa$ Ig light chain. 


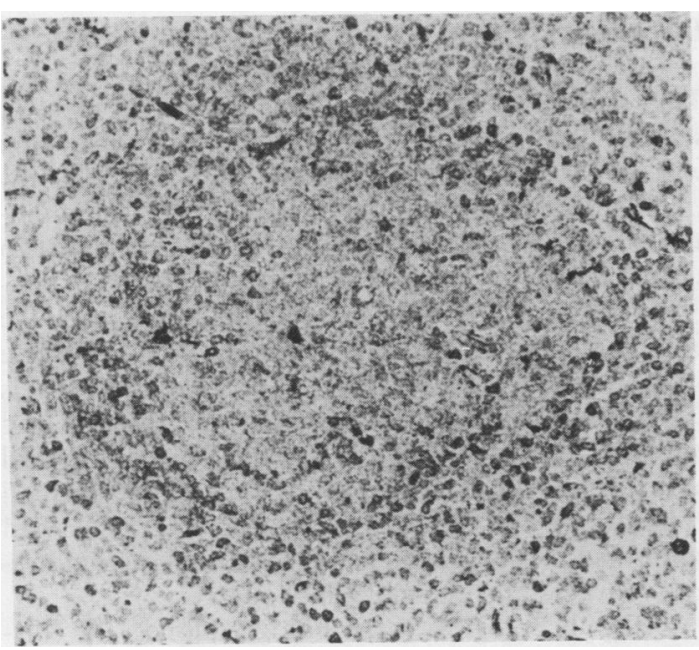

Fig. 3 Nodule of CLL with strong staining of most cells for Ig. Immunogold-silver staining technique for $\kappa$ Ig light chain.

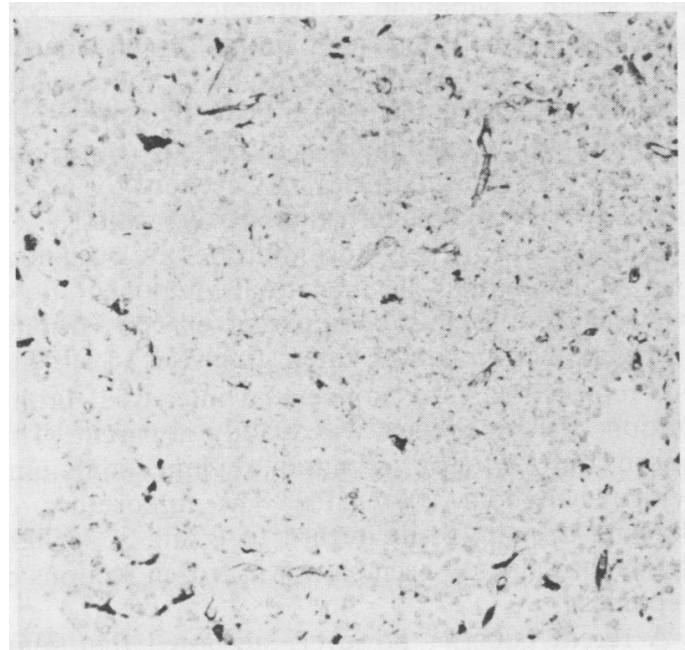

Fig. 4 Same nodule as in Fig. 3, but at a deeper level through tissue. Cells in aggregates are negative for $\mathrm{s}$ Ig. Some blood vessels stain positively. There is no evidence of dendritic reticulum cells using this method. Immunogold-silver staining technique for $\lambda$ Ig light chain.

occasional cells expressing intense activity for $\kappa$ and $\lambda$ cytoplasmic immunoglobulin $\left.{ }_{c} \mathrm{Ig}\right)$ light chains were present, both in the benign lymphoid aggregates and the intervening marrow spaces.

Furthermore, in the centres of the benign lymphoid aggregates, with or without secondary follicle centres, fine meshlike structures were observed lying between the follicle centre cells. These expressed both $\kappa$ and $\lambda$ ${ }_{s}$ Ig (Fig. 2).

FOCAL CHRONIC LYMPHOCYTIC LEUKAEMIA In all cases the appearances were uniform and consis-

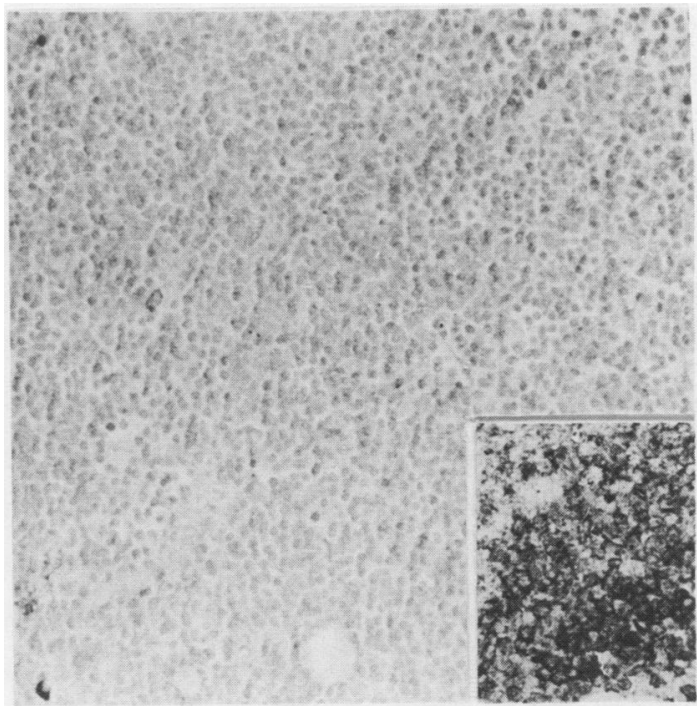

Fig. 5 Typical deposit of CLL lacking staining for $\lambda_{\mathrm{s}} \mathrm{Ig}$. Nodule did, however, stain positively for $\kappa_{\mathrm{s}}$ Ig. Inset: part of same nodule stained for $\kappa_{\mathrm{s}} I g$. Immunogold-silver staining technique for $\lambda$ Ig light chain (Inset for $\kappa$ chain).

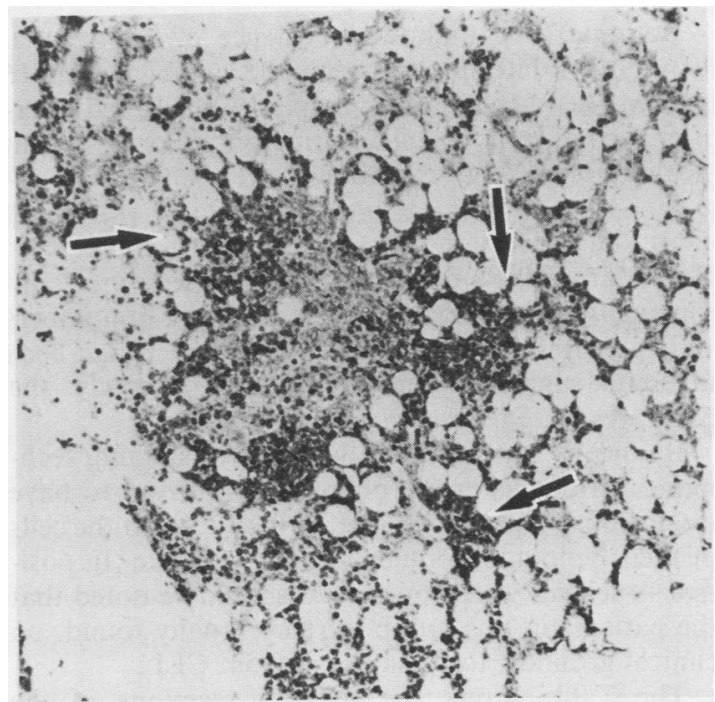

Fig. 6 Nodule of CLL with "overspill" of cells into internodular areas (arrows). Immunogold-silver staining technique for $\lambda$ chain.

tent. The infiltrating cells were monomorphic and possessed rounded regular small nuclei and little or no appreciable cytoplasm. Follicle proliferation centres were not seen.

The immunogold-silver staining technique showed that most cells in these nodules bore either $\kappa$ or $\lambda_{s} \mathrm{Ig}$ light chain but not both (Figs. 3, 4, and 5). Branching 


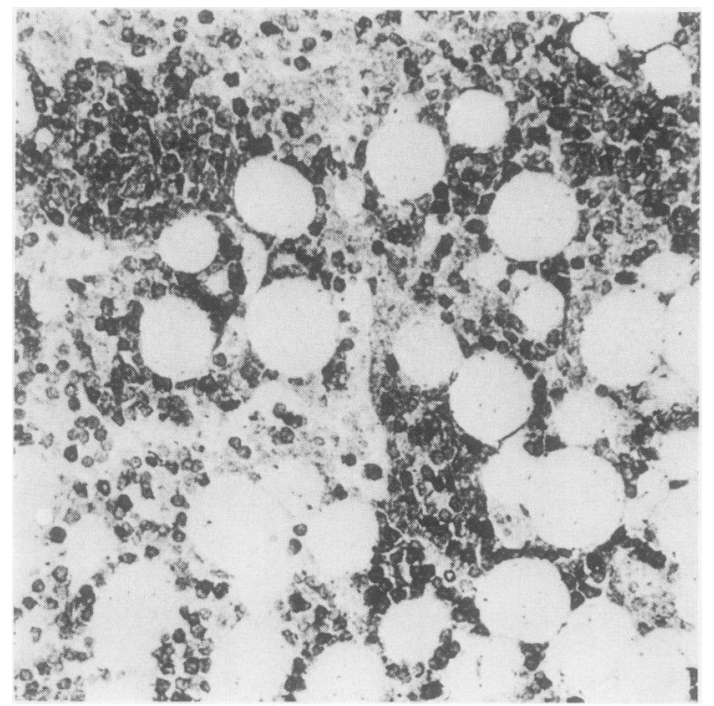

Fig. 7 Part of field in Fig. 6, showing "overspill" of positive cells at higher magnification. Immunogold-silver staining technique for $\lambda$ chain.

meshwork structures were not observed in these nodules.

Staining for ${ }_{s} \mathrm{Ig}$ showed evidence of monoclonal lymphoid infiltration between the aggregates in 16 specimens. In these a rather sparsely scattered internodular infiltrate of cells bearing only $\kappa$ or $\lambda_{\mathrm{s}} \mathrm{Ig}$ (but not monotypic ${ }_{\mathrm{c}} \mathrm{Ig}$ ) was observed (Figs. 6 and 7 ).

\section{INDETERMINATE SPECIMENS}

Secondary follicle formation was not seen in a convincing form in these specimens. The lack of numbers of aggregates and their small size, however, had been taken to suggest a benign nature. Conversely, the infiltrates were relatively monomorphic.

By means of the immunogold-silver staining technique, all of this group of trephines proved to have monoclonal expression of either $\kappa$ or $\lambda_{s}$ Ig on the cells of their lymphoid nodules. Again, meshlike ${ }_{s}$ Ig positive structures were not seen. It should be noted that the patients in this group were eventually found, on clinical grounds, to be suffering from CLL.

The Table shows the staining reactions of the aggregate cells for $\kappa$ and $\lambda_{s}$ Ig light chains.

In all cases the diagnosis of B-CLL had been confirmed by peripheral lymphocyte marker studies.

\section{Discussion}

This study has shown that the immunogold-silver staining technique can readily be applied to paraffin wax sections of formol-acetic acid fixed bone marrow trephines. The simple combination of immunostaining for $\kappa$ and $\lambda$ light chains alone is sufficient to show clonality and thus malignancy in focal lymphoid aggregates by virtue of the ability of the immunogold-silver staining method to show surface Ig on lymphoid cells. The need for frozen sections is thus avoided, providing improved morphology and retrospective study. The potentially tautological nature of a study such as this is excluded as all patients in the CLL group, for example, had confirmed disease on the basis of peripheral lymphocyte markers and clinical behaviour.

Using histological morphology alone, the distinction between benign lymphoid aggregates and CLL is, indeed, often most difficult. Four main types of benign lymphoid aggregates have been described $^{78}$ : small clusters of lymphoid cells; sharply outlined and well defined nodules; and nodules with follicle centres. The distinction between sharply outlined and well defined nodules is, perhaps, uncertain. Nodules with follicle centres may account for as few as only $5 \%$ of all benign lymphoid aggregates.

In the case of B-CLL most infiltrates are both nodular and interstitial. The interstitial component, however, may not readily be apparent on conventional histological examination. Interestingly, in 16 of 20 of our reported cases a monoclonal interstitial proliferation of lymphocytes was readily apparent. This was in contrast to the findings in trephines containing benign lymphoid aggregates. The importance of showing clonality, with regard to $\kappa$ and $\lambda_{\mathrm{s}} \mathrm{Ig}$ light chains, has already been shown in frozen sections of trephines. ${ }^{4}$

A recent paper, ${ }^{10}$ however, suggested that some specimens containing benign lymphoid aggregates may show monoclonal ${ }_{s}$ Ig expression on the cells of the aggregates. The authors emphasise that this finding could represent a preleukaemic picture. Notwithstanding these findings, our series showed a well defined correlation between malignancy, as assessed on obvious clinical grounds and by means of peripheral cell markers, and monoclonality in the lymphoid aggregates in the trephines. Furthermore, the speci-

Table Staining for benign lymphoid aggregates, chronic lymphocytic leukaemic nodules, and indeterminate aggregates for $\kappa$ and $\lambda$ light chains, using immunogold-silver staining

\begin{tabular}{llll}
\hline Type of aggregate & No of specimens & Positively stained & Final diagnosis \\
\hline Benign lymphoid aggregate & 12 & $\kappa$ and $\lambda$ & Benign lymphoid aggregate \\
Chronic lymphocytic leukaemia & 14 & $\kappa$ & Chronic lymphocytic leukaemia \\
Indeterminate* & 6 & $\kappa$ & Chronic lymphocytic leukaemia
\end{tabular}

*Uncertain on histological grounds. Diagnosis subsequently established clinicopathologically. 
mens of indeterminate histology, shown to possess monoclonality, subsequently proved to have B-CLL.

Another morphological guideline, taken to suggest that lymphoid aggregates are benign, is that of the presence of secondary follicle centres. This is most unreliable, however, as these are observed only in a minority of benign lymphoid aggregates. ${ }^{78}$ Indeed, follicle centres may also occur in malignant lymphoid aggregates (although probably not in B-CLL per se).

Immunohistochemical studies of paraffin sections of bone marrow trephines have been fairly neglected in the past despite their great diagnostic potential. Recently, however, several studies have used immunoperoxidase methodology to show plasma cells by virtue of their ${ }_{c} \mathrm{Ig}$ content ${ }^{6}$ and normoblasts by means of staining for haemoglobin. ${ }^{11}$ Similarly, megakaryocytes have been shown in trephines using an immunohistochemical sequence for factor VIII related antigen, ${ }^{1213}$ and granulocyte series cells have been shown by staining for leucocyte elastase ${ }^{14}$ and cathepsin G. ${ }^{15}$

The immunogold-silver staining technique has proved to be highly successful for showing B cell neoplasms in paraffin sections. ${ }^{5}$ This seems to result from the great sensitivity of the method, which enables the detection of ${ }_{s} \mathrm{Ig}$ in such specimens. Recently, the immunogold-silver staining method was used to show surface markers on peripheral blood lymphocytes in cytocentrifuge preparations, using OKT3, OKT4, OKT8, and OK 1al. ${ }^{16}$

The meshwork of activity for ${ }_{s} \operatorname{Ig} \kappa$ and $\lambda$ chains in the centres of benign lymphoid aggregates presumably represents adsorbed Ig on dendritic reticulum cells. These cells, which are present in normal and reactive follicle centres, possess surface Igs which may be shown (with difficulty) by immunoperoxidase techniques applied to paraffin sections. ${ }^{1718}$ Notably, although the immunogold-silver staining method for $\kappa$ and $\lambda$ chains showed these cells in benign lymphoid aggregates, none was seen in the CLL structures. This no doubt reflects previous findings that dendritic reticulum cells in malignant follicles, although present, do not display ${ }_{s}$ Ig activity. ${ }^{1920}$ Dendritic reticulum cells have also been shown to be present in malignant lymphoid nodules in frozen sections of marrow trephines by a monoclonal antibody (RFD 3). ${ }^{19}$ Thus the virtual or total absence of ${ }_{s}$ Ig bearing dendritic reticulum cells may provide a useful distinguishing feature of malignant in contrast to benign lymphoid aggregates in the marrow. To show this difference convincingly it may be necessary to use immunogold-silver staining methodology.

We thank Mrs V Garland for typing the manuscript and the West Midlands Regional Health Authority Research Committee for their generous financial sup- port. We are also indebted to $\mathrm{Mr} \mathrm{MJ}$ Chard for skilful photographic help.

\section{References}

${ }^{1}$ Krause JR. Bone marrow biopsy. Edinburgh: Churchill Livingstone, 1981.

${ }^{2}$ Frisch B, Lewis SM, Burkhardt R, Bartl R. Biopsy pathology of bone and bone marrow. London: Chapman and Hall, 1985:182.

${ }^{3}$ Warnke R, Pederson M, Williams C, Levy R. A study of lymphoproliferative diseases comparing immunofluorescence with immunohistochemistry. Am J Clin Pathol 1978;70:867-75.

${ }^{4}$ Pizzolo G, Chilosi M, Getto GL, Fiore-Donati L, Janossy G. Immuno-histological analysis of bone marrow involvement in lympho-proliferative disorders. Br J Haematol 1982;50:95-100.

${ }^{5}$ Holgate CS, Jackson P, Lauder I, Cowen PN, Bird CC. Surface membrane staining of immunoglobulins in paraffin sections of non-Hodgkin's lymphoma using immunogold-silver staining technique. J Clin Pathol 1983;36:742-6.

${ }^{6}$ Crocker J, Curran RC. Quantitative study of the immunoglobulincontaining cells in trephine samples of bone marrow. $J$ Clin Pathol 1981;34:1080-2.

${ }^{7}$ Hashimoto M, Masanori H, Tsukasa S. Lymphoid nodules in human bone marrow. Acta Pathologica Japan 1957;7:33-52.

${ }^{8}$ Rywlin AM, Ortega RS, Dominguez CJ. Lymphoid nodules of bone marrow, normal and abnormal. Blood 1974;43:389-400.

${ }^{9}$ Frisch B, Lewis SM, Burkhardt R, Bartl R. Biopsy pathology of bone and bone marrow. London: Chapman and Hall, 1985:188.

${ }^{10}$ Levy N, Nelson J, Meyer P, Lukes RJ, Parker JW. Reactive lymphoid hyperplasia with single class (monoclonal) surface immunoglobulin. Am J Clin Pathol 1983;80:300-8.

${ }^{11}$ Crocker J, Gyde OHB, Jenkins R. Demonstration of normoblasts in tissue sections by means of an immunohistochemical technique for haemoglobin. J Clin Pathol 1984;37:1312-3.

${ }^{12}$ Crocker J, Smith PJ. Value of factor VIII-related antigen as a means of demonstrating extramedullary megakaryopoiesis. $J$ Clin Pathol 1984;37:834-5.

${ }^{13}$ Crocker J, Smith PJ. Value of factor VIII-related antigen as a means of demonstrating extramedullary haemopoiesis. J Clin Pathol 1985;38:355.

${ }^{14}$ Crocker J, Jenkins R, Burnett D. Immunohistochemical demonstration of leucocyte elastase in human tissues. J Clin Pathol 1984;37:1114-8.

${ }^{15}$ Crocker J, Jenkins R, Burnett D. Immunohistochemical localisation of cathepsin G in human tissues. Am J Surg Pathol 1985;9:338-43.

${ }^{16}$ Romasco F, Rosenberg J, Wybran J. An immunogold silver staining method for the light microscopic analysis of blood lymphocyte subsets with monoclonal antibodies. Am J Clin Pathol 1985;84:307-16.

${ }^{17}$ Curran RC, Gregory J, Jones EL. The distribution of immunoglobulin and other plasma proteins in human reactive lymph nodes. J Pathol 1982;37:307-22.

${ }^{18}$ Crocker J, Jenkins R. An immunohistochemical study of branchial cysts. J Clin Pathol 1985;38:784-90.

${ }^{19}$ Chilosi M, Pizzolo G, Caligaris-Cappio F, et al. Immunohistochemical demonstration of follicular dendritic cells in bone marrow involvement of B-cell chronic lymphocytic leukaemia. Cancer 1985;56:328-32.

${ }^{20}$ Stein H, Gerdes J, Mason DY. The normal and malignant germinal centre. Clin Haematol 1982;11:531-58.

Requests for reprints to: Dr J Crocker, Department of Histopathology, East Birmingham Hospital, Bordesley Green East, Birmingham B9 5ST, England. 